(Mathematical Cognition, 1996, Vol.2, 113-135)

\title{
Estimation Strategies of Four Groups
}

\author{
ANN DOWKER
}

University of Oxford

AMANDA FLOOD

Christ Church, Oxford

\author{
HELEN GRIFFITHS \\ University College, Oxford
}

LOUISE HARRISS

St. Hilda's College, Oxford

LISA HOOK

Lincoln College, Oxford 


\begin{abstract}
Forty-four academic pure mathematicians, forty-four accountants, forty-four psychology students and forty-four English students were given Levine's (1982) computational estimation task, which involved mentally estimating the products and quotients of 20 multiplication and division problems and describing their strategies. The mathematicians were the most accurate estimators, and the English students the least accurate, with psychology students and accountants obtaining similar scores intermediate between the other groups. All groups demonstrated an impressively versatile use of appropriate strategies. The mathematicians and accountants used significantly larger numbers of appropriate strategies than the other groups, and strongly resembled one another in this respect, despite the significantly greater accuracy of the mathematicians. All of the non-mathematician groups used significantly larger numbers of inappropriate strategies than did the mathematicians. We discuss (1) the implications for cognitive psychology of the great variability of strategy use in an apparently simple task; and (2) the relationship between people's mathematical knowledge and experience and their estimation accuracy and strategy variability.
\end{abstract}




\section{INTRODUCTION}

This study concerns the estimation strategies of four professional/student groups: academic mathematicians, professional accountants, psychology students and English students. Computational estimation is defined as giving approximate answers to arithmetic problems, without or before actually doing the calculations. As well as being a skill of some practical importance, it provides useful information about arithmetical cognition, in that it makes it possible to separate certain components of arithmetic that are particularly important in exact calculation (arithmetical fact retrieval; detailed memory for arithmetical procedures) from others that are particularly important in estimation. The latter would seem to include, in particular, (1) the approximate understanding of quantities; and (2) ability to devise and use strategies for manipulating numbers, when no one specific procedure is clearly required. The main emphasis of this study is on this second component, with a focus on both the nature and variability of the strategies used.

Despite the popular view (cf. Gill, 1993) that a mathematical problem usually has only one unique optimal solution method, the versatile use of standard and nonstandard strategies can also be observed in exact calculation, of very simple sums by young children (Siegler and Jenkins, 1989), of more difficult problems by older children and adults (Smith, 1995) and even of quite simple sums by adults (Lefevre, Sadesky and Bisanz, in press; Lefevre, Bisanz, Hubbard, Buffone, Greenham and Sadesky, submitted). Nevertheless, the fact that estimation is an open-ended task, where problems usually do not have a single correct answer, makes it a particularly useful tool for investigating the extent of flexibility, versatility and variation of strategy use.

There have already been a number of studies dealing with computational estimation by people of various levels of arithmetical experience and ability, ranging from very young children to adults. Baroody $(1989,1990)$ and Dowker (1989) have studied young children's addition estimates and found that they do use some estimation strategies, which, while often 
limited and even inappropriate, reveal an understanding of certain properties of number. Some adolescents and adults show considerable skill in estimation (Reys, Rybolt, Bestgen and Wyatt, 1982), usually without having received explicit instruction in estimation. Many, however, do not (e.g. Hiebert, 1984). Levine (1982) gave 89 American college students, who were not majoring in mathematics, a task involving estimating the approximate solutions to a set of multiplication and division problems, and studied both the accuracy of their estimates and the strategies used. She found that the general standard was quite low, with a mean score of 25.9 out of a potential maximum of 60 . Moreover, many subjects appeared to use only a narrow range of strategies.

By contrast, it has recently been noted that computational and quantitative estimation can sometimes be relatively good in patients whose knowledge of arithmetical facts is seriously impaired (Warrington, 1982) or even virtually absent (Dehaene \& Cohen, 1991). It has been suggested (Dehaene \& Cohen, 1991; Dehaene, 1992) that there is a system for comparison and approximation of numerical quantities that is quite separable from exact calculation; and that is indeed present to some extent in preverbal infants and some non-human animal species. In many cases, however, the estimation ability of such patients does not appear to depend totally on such a separate mechanism, but is a result of their retaining a good conceptual understanding of arithmetical principles, which enable them to devise strategies for deriving answers (sometimes exact, but often approximate) to problems that their memory deficits prevent them from solving in standard ways (Warrington, 1982; Sokol \& McCloskey, 1991; Hittmair-Delazer, Semenza \& Denes, 1994; Hittmair-Delazer, Sailer \& Benke, 1995).

It could be argued, however, that most of the studies mentioned above show only that non-school taught strategy use and arithmetical estimation are important to people who do not have access to school taught strategies: in particular, those who have had their access to arithmetical knowledge disrupted through brain damage, and perhaps also children who have 
not yet completed their school instruction. Do schooled adults have any need or desire to use nonstandard arithmetical strategies? Will they see any point in estimating? If they do estimate, is this simply because they may have in fact been given some school instruction in estimation; and will they only use school-type estimation strategies?

A study of the estimation strategies of 44 professional academic mathematicians has been carried out (Dowker, 1992), and suggests that such subjects do indeed use an extremely wide variety of standard and, more often, nonstandard estimation strategies. The focus of interest in that study was the general nature of strategy use in computational estimation by people with exceptional number sense, rather than the effects of specific expertise in the use of specific strategies. Levine's (1982) task was used for this purpose.

In that study the mathematicians were shown to be very accurate estimators, though only a few obtained perfect scores (see Table 1). The most striking result, however, was the great variability of strategy use by the mathematicians. Many different appropriate strategies were used for each problem; each individual mathematician used several different broad strategy types per protocol; and when 18 were retested after several months, they frequently used totally different strategies for the same problems. This contradicted the popular view (see above) that all mathematical problems have unique optimal solution methods. An obvious question arising from this finding is whether such variability is specific to mathematicians, or whether it is a general characteristic of responses to an open ended problem solving task of this nature.

To investigate this issue, the present study compares four groups: the mathematicians of Dowker's (1992) study, accountants, psychology students and English students. All subjects had studied mathematics at least until the age of 16, and had thus been exposed extensively to arithmetic problems at least as difficult as those in the estimation task. However, there were marked group differences as regards experience with number. The mathematicians had extensive experience with and interest in the properties of and 
relationships between numbers, though they were not especially versed in estimation or calculation. The accountants had more experience than the mathematicians in selecting, planning and carrying out calculations in practical situations, but less theoretical interest in numerical properties and relationships. Neither the psychology students nor the English students had extensive professional interest in number as such, but the psychology students had considerably more mathematical background (e.g. statistics) than the English students. Therefore the comparison of the groups makes it possible not only to study the nature and variability of estimation strategy use, but also to investigate the relationship between these and subjects' general mathematical experience.

A priori, it would appear that people who have a wide knowledge of a domain are potentially more versatile in their strategy use: they have access to a greater variety of strategies and also may be better able to perceive relationships and problem characteristics which facilitate the construction of new strategies. A number of people have proposed a positive relationship between mathematical ability and mathematical strategy variability (Krutetskii, 1981; Haylock, 1985); and Baroody (personal communication) has suggested that children's arithmetical strategies may become more differentiated with increasing age and knowledge. On the other hand, it could be suggested (and would, for example accord with some production system theories; cf. Linville and Clark, 1989) that mathematically knowledgeable people might be less variable than those with less mathematical knowledge, because their strategy use is more automatized, or because they are more aware of which strategies are maximally efficient for a given problem, and use these.

Two theoretical viewpoints are central to the present study:

(1) Strategy variability is indeed a fundamental characteristic of human mathematical (and much non- mathematical) cognition. The prediction arising from this is that all the groups studied should show considerable variability. 
(2) At least as regards arithmetical estimation and some related domains, there may be a U-shaped relationship between knowledge/understanding and variety of strategy use. Novices - notably children (Ginsburg, 1977; Dowker, 1989; Siegler and Jenkins, 1989) - may use a large variety of strategies (appropriate and inappropriate), because they have not yet acquired a small set of well-learned strategies. People with extensive knowledge and ability may use a variety of strategies (mostly appropriate), because they have access not only to a large number of strategies but to principles and relationships that facilitate the invention of new strategies. People at an intermediate level of knowledge are more likely to have mastered a limited number of strategies and to rely on these. This hypothesis may be compared with Sternberg's (1988, pp. 137-138) inverted U-shaped hypothesis, and with Karmiloff-Smith's (1990) U-shaped hypothesis proposed on somewhat different theoretical grounds with regard to children's development.

The present study included no novices: despite important group differences, all subjects had extensive experience with numerical tasks. The prediction was therefore made that, in the present study, mathematical knowledge should be positively related to strategy variability. It was also predicted that people with an interest in numerical relationships for their own sake (mathematicians) would be more variable than those whose experience primarily involves arithmetical problem solving for the purpose of achieving defined practical goals (accountants). Thus the predicted rank order of groups in this study, with regard to strategy variability, was: mathematicians > accountants > psychology students > English students.

In discussing strategy variability, it is important to distinguish here between -individual variability (e.g. number of specific strategies used by different individuals for the same problem) and within-individual variability (e.g. the number of problems for which an 
individual uses different strategies if tested more than once, and the number of strategy types used per protocol). Both types of variability provide an important indication of cognitive flexibility. Within-individual variability provides a purer measure of an internal predilection for strategy variation, as it is less likely to be affected by extraneous factors (e.g. different schools teaching different strategies). It was predicted that similar group differences would be demonstrated for both types of variability. It was also predicted that, within each group, there would be a positive correlation between estimation accuracy and measures of within-individual variability.

The particular aspect of between-strategy variability investigated in this study was number of specific strategies used for each problem.

The aspects of within-individual variability investigated were:

(1) number of strategy types used by each person. These were classified according to a scheme based on that of Levine (1982) but modified to take into account of strategies that were used with some frequency by the subjects in this study. The strategy types are listed on pp.9-11).

(2) the nature of these strategy types. Strategy types may be divided into two broad classes. Class 1 involves the application of all purpose techniques to particular problems. These include algorithmic and rounding strategies (see pp.9-10), and have the following characteristics: they are likely to be taught in school, and in theory they can be applied to any problem of the general type studied here without reference to the particular characteristics of a specific problem. Class 2 involves attention to the particular numbers in a problem, and/or the relationships between them. Such strategies include factorization, use of fractional relationships, and the use of known and nicer numbers (see p.10). While this is not in the strict sense a variability measure, it is closely related in that the frequent use of Class 2 strategies would imply 
a flexible adaptation of strategy to problem type, without reliance on standard procedures;

(3) tendency to use different strategies for the same problems on repeated testing. While this was probably the most refined and discriminating measure, it was unfortunately the case that only some of the mathematicians and psychology students, and almost none of the other subjects, were available for retesting. Therefore the only analysis carried out with regard to this measure was a comparison between mathematicians and psychology students: greater test/retest variation was predicted for the mathematicians.

\section{METHOD}

Forty-four professional 'pure' mathematicians, forty-four professional accountants, forty-four psychology students and forty-four English students took part in this study. The performance of the mathematicians has already been described (Dowker, 1992), but without any comparisons with other groups.

Most of the mathematicians were at least at mid-career and were established at various universities in the United Kingdom and North America. Many of them were professors and eight were fellows of the Royal Society or of similar organisations abroad. Thirty-nine were men and five were women. Exact ages were not available for all subjects, but ranged from 25 to $70+$ with a median in the late 40 s.

The accountants came from a wider range of educational backgrounds than the other groups. Twenty had reached only 'O' level standard (examination taken at age 16) in mathematics, twenty had reached 'A' level (examination taken at age 18), and four had degrees in mathematics. With regard to general educational background, thirteen were educated to 'O' level standard only, seven to 'A' level, and twenty-four to degree level. 
Thirty-three were men and eleven were women. Ages ranged from 21 to 65 , with a median of 33.

Forty of the psychology students were Oxford undergraduates at the time of the study, two had recently graduated from Oxford, and two were postgraduate students (one at Oxford; one at London). Twenty-two were men and twenty-two were women. All had mathematics 'O' level or the equivalent and twenty-four had mathematics 'A' level. The age range was from 18 to 30 , with an approximate median of 20 .

Thirty-three of the English students were Oxford undergraduates at the time of the study, three had recently graduated from Oxford, two had recently graduated from Cambridge, and seven were postgraduate students (six at the University of Kent at Canterbury; one at UCLA). Nineteen were men and twenty-five were women. All had mathematics 'O' level or the equivalent, but only one had mathematics 'A' level. Ages ranged from 18 to approximately 35, with an approximate median of 20.

The basic procedure was that used by Levine (1982) in her study of American college students who were not majoring in mathematics. The task used was Levine's Test of Estimation Ability, which involved mentally estimating the products and quotients of 10 multiplication and 10 division problems. The items were presented one at a time in random order. Each problem was simultaneously presented orally and in written form. Instructions were, "I am going to give you some multiplication and division problems, and I would like you not to calculate the answers exactly, but to estimate the answers. I would like you not to use pencil and paper, but to think aloud while you are estimating, and describe the strategies that you use. My main interest is in what strategies people use, rather than how good or bad they are at estimating". There was no time limit. Each problem remained visible to the subject as long as needed. Their responses were recorded on a tape-recorder.

The items used in the task were:
(1) $76 \times 89$
(11) $9208 \div 32$ 

(2) $93 \times 18$
(12) $4645 \div 18$
(3) $145 \times 37$
(13) $7858 \div 51$
(4) $824 \times 26$
(14) $25410 \div 65$
(5) $187.5 \times 0.06$
(15) $648.9 \div 22.4$
(6) $482 \times 51.2$
(16) $546 \div 33.5$
(7) $64.6 \times 0.16$
(17) $1292.8 \div 71.2$
(8) $424 \times 0.76$
(18) $66 \div 0.96$
(9) $12.6 \times 11.4$
(19) $943 \div 0.48$
(10) $0.47 \times 0.26$
(20) $0.76 \div 0.89$

Levine's scoring system (1982, p. 350) was used. If an estimate was no more than $10 \%$ away from the exact product or quotient, three points were scored. If the percentage of error was greater than $10 \%$ but no more than $20 \%$, two points were scored. If the percentage of error was greater than $20 \%$ but no more than $30 \%$, one point was scored. Zero points were scored if the percentage of error was greater than $30 \%$.

The types of strategies used were classified according to a scheme based on that used by Levine (1982, pp. 352-353), but modified to take account of strategies that were used with some frequency by the subjects in this study, but rarely or not at all by Levine's subjects. The scheme included the eight following strategy types, which are further divided into two main classes (see p.6, N.2): Class 1 comprising 'all-purpose', potentially school-taught strategies, and $\underline{\text { Class } 2}$ comprising strategies more specifically adapted to the features of a particular problem:

\section{Class 1:}

1. Rounding Two Numbers (Levine's Type 3). This strategy involves rounding each of the two numbers either upward or downward to the nearest multiple of some non-zero 
power of 10 (having fewer significant digits than the given number) for example, rounding the problem $4645 \div 18$ to $4640 \div 20$ giving 232 , or $4600 \div 20$ giving 230 . If the two numbers are both less than 20 , then taking the decimal point off counts as rounding, for example converting the problem $12.6 \times 11.4$ to $12 \times 11$.

2. Rounding One Number (Levine's Type 4). One of the two numbers is rounded as described above. No substantive change is made in the second number. Examples include converting the problem $76 \times 89$ to 76 × 90 and converting the problem $93 \times 18$ to $93 \times 20$.

3. Proceeding Algorithmically (Levine's Type 8.) In this strategy type, any form of a standard algorithm is used to calculate roughly, estimate and then combine all partial products or quotients: for example, responding to the problem $424 \times 0.76$ by approximate long multiplication of 424 by 70 and then 6 to give about 32,000, followed by division by 100 to give 320 .

\section{Class 2:}

1. Use of Fractions, (Levine's Type 1). In this strategy, fractional relationships are used: for example, restating the problem $824 \times 26$ as about $824 \times 25=825 \times 0.25 \times 100=$ $(824 \times 1 / 4) \times 100=(824 \div 4) \times 100=20,600$; or restating the problem $64.6 \times 0.16$ as $66 \times 1 / 6=11$.

2. Using Known or Nice-Numbers. This strategy involves converting the numbers in the problem to nicer, more compatible numbers in ways not subsumed under other strategy types: for example converting $25410 \div 65$ to $24000 \div 60$, making division (giving the answer 400) much easier. This strategy differs from rounding strategies in that at least one of the numbers in the problem is converted to something other than the nearest multiple of 10 .

5. Factorization. This strategy type involved breaking up numbers into component factors, or dividing the two numbers in the problem through by a common factor: for 
example, estimating $550 \div 33$ as " $(550 \div 11) \div(33 \div 11)$, which is $50 \div 3$, which is about 16".

7. Distributivity. In this strategy type, which subsumes Levine's Incomplete Partial Products, strategy, the distributivity principle of multiplication is used: for example, treating the problem $76 \times 89$ as about $76 \times 90=76 \times(100-10)=(76 \times 100)-(76 \times$ 10) $=7600-760=$ about 6800 .

8. Other categories, including rare and idiosyncratic responses and those that were only used for one or two problems. The most important of these are:

(A) Recognition that the division of a number by a fraction fairly close to 1 will result in a number just a little greater than the original number: for example that $0.76 \div 0.89$ will be a little more than 0.76 .

(B) Use of the rule that $(a+b)(a-b)=a^{2}-b^{2}$, for example, giving the exact answer to the problem $12.6 \times 11.4$ by converting it to: $(12+0.6) \times(12-0.6)=$ $12^{2}-(0.6)^{2}=144-0.36=143.64$

All responses were classified by the first author according to the above classification scheme. Independent raters classified the strategies used in the protocols of four randomly-selected subjects in each group (16 subjects; 320 responses. The protocols of the four mathematicians were rated by one rater, and the other 12 protocols by another.) Interrater agreement was $94 \%$.

Eighteen of the 44 mathematicians and twenty of the 44 psychology students were given the same task again after an interval of 6 to 9 months, and their strategies on the two occasions were compared. It was unfortunately not possible to retest the accountants or the English students. 


\section{$\underline{\text { Accuracy }}$}

The means, standard deviations and ranges of accuracy scores are shown in Table 1.

\section{Table 1 about here}

One-way analyses of variance revealed significant group differences, as shown in Table 2.

\section{Table 2 about here}

The 18 mathematicians who were subsequently retested obtained a mean score on initial testing of 53.28 (s.d. 5.28) and an identical mean score on retesting of 53.28 (s.d. 6.57). The 20 psychology students who were subsequently retested obtained a mean initial score of 43.82 (s.d. 8.92) and a mean retest score of 45.47 (s.d. 7.96). A two-tailed matched-pairs t-test showed no significant difference between the psychology students' initial and retest scores $(t=-0.86 ; \mathrm{df}=19 ; \mathrm{p}>0.05)$.

\section{$\underline{\text { Strategy types }}$}

Table 3 about here

Table 4 gives the chi-square values for comparisons between each pair of groups as regards the frequency of use of each strategy type.

Table 4 about here

\section{Strategy types: number and classification in individual protocols}


Means, ranges and standard deviations of (a) number of strategy types per protocol and (b) number of responses per protocol involving Class 2 strategies are shown in Table 5. Table 6 gives the group comparisons for these measures. It can be seen that mathematicians used more strategy types per protocol, and also gave more responses involving Class 2 strategies, than did the other groups.

Table 5 about here

Table 6 about here

\section{$\underline{\text { Specific strategies }}$}

Within each strategy type, as applied to a given problem, it was usually possible to identify a number of specific strategies. Figure 1 gives all the 26 specific strategies that were used for the problem 4645 - 18 (the second-time protocols of people who took the test twice are not considered here).

Figure 1 about here

A distinction should be made between appropriate and inappropriate specific strategies. An appropriate strategy is defined here as one which (1) is consistent with mathematical principles and (2) if practised without a calculation error, would result in an estimate within $20 \%$ of the correct answer.

Once these two criteria were met, a strategy was classed as appropriate regardless of precise degree of accuracy, elegance, simplicity, mathematical sophistication or the presence or absence of calculation errors in a particular instance. 
The mean number of appropriate specific strategies used per problem by each group was 12.45 (s.d. 3.66) for the mathematicians, 12.75 (s.d. 3.67) for the accountants, 10.45 (s.d. 3.28) for the psychology students, and 10.15 (s.d. 2.25) for the English students. The mean number of inappropriate specific strategies used per problem by each group was 1.4 (s.d. 1.53) for the mathematicians, 2.15 (s.d. 1.35) for the accountants, 2.8 (s.d. 2.375) for the psychology students, and 2.95 (s.d. 2.2) for the English students. Table 7 gives the t-values for comparisons between the groups on numbers of appropriate and inappropriate strategies used per problem.

Table 7 about here

\section{Is accuracy related to variability?}

Pearson correlation coefficients were computed within each group between score and

(a) number of strategy types used per protocol and (b) number of Class 2 responses per protocol. 


\section{Effect of educational background: the accountants}

The accountants' scores and numbers of strategy types were analysed with respect to the educational background measures summarised on p7. Mean scores were 38.76 (s.d. 9.27) for those with only mathematics O Level; 44 (s.d. 7.78) for those with mathematics A Level; and 46.75 (s.d. 7.5) for those with mathematics degrees. They were 38.89 (s.d. 7.17) for those generally educated only to O-Level standard, 37.33 (s.d. 10.97) for those with A Levels, and 44.86 (s.d. 9.78) for those with degrees.

Mean numbers of strategy types per subject were 5.5 (s.d. 0.72) for those with only mathematics O Level, 5.67 (s.d. 1.0) for those with mathematics A Level, and 5.33 (s.d. 0.89) for those with degrees in mathematics. They were 5.46 (s.d. 0.88) for those generally educated only to O-Level standard, 5.56 (s.d. 0.88) for those educated to A-Level standard, and 5.68 (s.d. 0.82) for those with degrees.

There was no significant effect of mathematics education level $(F(2,41)=3.21 ; \underline{p}$ n.s. $)$ or general education level $(\mathrm{F}(2,41)=3.12)$ on score. There was also no significant effect of mathematics education level $(\mathrm{F}(2,41)=3.22$; $\mathrm{p}$ n.s. $)$ or general education level $(\mathrm{F}(2,41)=$ $3.22 ; \mathrm{p}$ n.s.) on the number of strategy types used per subject.

\section{Effects of gender:}

As shown in Table 9, gender differences were investigated within each group separately, except for the mathematicians, among whom there were too few women to make such an investigation appropriate. 
Among psychology students, males obtained higher scores and used Class 2 responses more often than did females. There were no significant gender differences in the other groups.

\section{Strategy variation on repeated testing:}

The mathematicians used different strategy types on the two testings for a mean of 9.17 out of the 20 problems (s.d. 2.2) and different specific strategies on the two testings for a mean of 13.6 out of the 20 problems (s.d. 2.47). The psychology students used different strategy types on the two testings for a mean of 7.45 problems (s.d. 2.74) and different specific strategies for a mean of 11.9 problems (s.d. 2.17). One-way analyses of variance showed significant differences between the mathematicians and the psychology students as regards strategy types $(\mathrm{F}(1,36)=4.46 ; \mathrm{p}<0.05)$ and as regards specific strategies $(\mathrm{F}(1,36)=$ $5.49 ; \mathrm{p}<0.05)$.

\section{DISCUSSION}

Probably the most predictable result of this study (Table 1) was that the mathematicians were the most accurate estimators, and the English students the least accurate, with accountants and psychology students intermediate between the other two groups. In the groups (mathematicians and psychology students) where some subjects were retested, there were no significant test/retest differences in accuracy. All groups, including the English students, achieved higher mean scores than Levine's non-mathematician college students, perhaps reflecting the selection criteria used in admitting the English students to their courses in the first place. The only aspect of these results that was slightly surprising was that the accountants were only as accurate as the psychology students. This could possibly be due to the fact that the average educational level of the accountants was lower than that of the other groups. However it should be noted that educational level did not significantly affect the accountants' estimation accuracy within their group (p.14). 
The most striking result of the study is that all groups showed both between-individual and within-individual variability to a very marked degree. All groups used a remarkably large number and variety of (mostly appropriate) strategies for every problem (p.13). Within-individual variability was also great, whether assessed by number of strategy types used (Table 5), test-retest variation (p.15); or the more indirect measure of number of Class 2 responses (Table 5).

Although great variability in this task was a characteristic of all groups, there were some highly significant group and individual differences. As predicted, these were mostly in the direction of greater variability being associated with greater arithmetical knowledge and experience.

The generally high level of variability and its implications will be discussed first before proceeding to the implications of group differences in strategy variability, and finally to the influence of both group and problem on preferred strategy types.

These results give rise to questions about the general extent and predictability of strategy variation in human cognition; about the extent to which such strategy variation is rule-governed; and about the role of nondeterminism in cognition in general and in creative or novel thought in particular. There is still no definite consensus as to the role played by nondeterminism in creative or novel cognition. For example, Johnson-Laird (1988) implies the necessity for some kind of randomness in the brain, while Boden (1991) does not ascribe an important role to randomness.

In view of the extreme variability found here, a central question is whether the variability of strategy choice, in a task even as small as the present one, is rule-governed in ways that would eventually become predictable if sufficiently many individuals were tested sufficiently many times. Is their strategy use rule-governed, but with the rules ultimately leading to what mathematicians term 'chaos' (see Stewart, 1989)? Is it essentially random? 
Or could it possibly be explained to some extent by certain non-serial-digital artificial intelligence models (cf. Bates and Elman, 1993)?

Several AI models of human reasoning have recently been developed, which incorporate some degree of non-determinism (e.g. Thagard, Holyoak, Nelson \& Gochfield, 1990; Hofstadter, 1984, 1995; Hofstadter, Mitchell \& French, 1987; Mitchell, 1994). Such models are designed to select concepts and/or strategies among a number of possibilities on the basis of probabilistic rather than completely deterministic rules, and to vary the level of determinism involved in such selection processes according to the situation or the stage that has been reached in solving a particular problem. Other relevant models include those devised by Lenat and Brown (1984) and Fajtlowicz (1988, 1989) to model mathematicicans' conjecturing and those that Siegler and his colleagues (Siegler and Jenkins 1989) are developing with regard to children's strategy choice in arithmetic.

Defays' $(1988,1990)$ computer program "Numbo" is probably the most relevant to the present study. It plays a game involving the construction of a given number (the 'target') from a given set of five other numbers (the 'bricks'), using the arithmetical operations of addition, multiplication and subtraction. It encodes a set of rote-learned number facts (e.g. multiplication tables); a set of "landmark numbers" such as 100 to which other numbers may be rounded if close in size; and a set of "salient numbers" and their decompositions such as perfect squares and square roots. These bear some resemblance to some of the number properties used in the strategies of the subjects in the present study: especially those included in the Rounding One Number, Rounding Two Numbers, and Known and Nicer Numbers strategy types. Numbo contains probabilistic rather than absolute rules for strategy selection and can use different strategies and give different responses to the same problem on different runs.

We suggest that there are certain situations where it may be particularly difficult to provide a deterministically predictive explanation for novel behaviour. At one extreme, the 
work of Goedel or Ramanujan presents a great challenge to any attempts at universal predictive theories of how creativity occurs. At the other extreme, as Perkins (1981, p. 173) has pointed out, "all of us are improvisers much of the time, in ways we take for granted. We all create appropriate behaviour spontaneously in conversation and other everyday situations". Such behaviour is partially dependent on stereotyped patterns, and is partially influenced by context, but it is also highly, possibly infinitely, variable. It is here suggested that the performance discussed in this paper comes to some extent into such a category of simple yet highly variable behaviour, and may thus paradoxically be more difficult to describe deterministically than some more complex forms of problem-solving.

\section{Group and individual differences in variability}

The results imply that human mathematical cognition involves great versatility; but they also support the hypothesis that this varies significantly with mathematical ability and knowledge. As predicted, most measures showed the mathematicians to be the most variable and the English students the least versatile, with the other groups in between. The mathematicians and accountants showed a similar high level of between-individual variability (Table 7), but the mathematicians outperformed all other groups to a striking degree on those measures of within-individual variability that were available for all groups: number of strategy types per protocol (Table 6), and, most strikingly, frequency of Class 2 strategy use (Table 6). They also showed significantly greater test-retest variation than the one available comparison group: psychology students (p.15). Moreover, though some of the variability measures used here had not been used by Levine (1982), all the groups in the present study appeared more variable in strategy use than her presumably less mathematically able subjects.

Not only were the more mathematically experienced groups more variable in their strategy use; but there was an overall tendency within groups (Table 8) for estimation accuracy to be associated with within-individual variability: better estimators used more 
strategy types per protocol and their responses were more likely to involve Class 2 strategies. These within-group relationships were most marked in the student groups, and were less marked in the more mathematically experienced groups. The latter finding may have been in part due to ceiling effects in accuracy and/or variability, though even in the most accurate group (mathematicians) there was a positive relationship between accuracy and Class 2 strategy use.

The findings are consistent either with a monotonic positive relationship between strategy variability and mathematical experience, or with the U-shaped relationship proposed in the Introduction. It would be necessary to test less arithmetically advanced subjects (e.g. schoolchildren) to determine whether the hypothesis of a U-shaped relationship is in fact correct.

We suggest that such U-curves are likely to describe the relationship between expertise and variability both in this domain and in many but by no means all other domains. They are more likely to be applicable to domains involving commonly-occurring, partially but not totally stereotyped activities, such as for instance conversation (cf. Perkins, 1981). They are less likely to be applicable to domains that involve either highly routinized rote-learned skills, for which expertise is likely to mean the highly automatized use of a single strategy, or highly specialized and instruction-dependent skills where novices may be for a significant period of time unable to attempt any strategies other than those which they are explicitly instructed to perform.

It is of course not possible to consider the relationship between arithmetical knowledge and strategy variability in isolation from three issues: (1) the type of knowledge and understanding being considered; (2) the nature and difficulty of problems being considered; and (3) whether one considers all strategies or only those which are appropriate.

\section{Type of knowledge and understanding}


It needs to be remembered that the present study was not intended to compare groups of people who were and were not 'expert' at tasks similar to the one presented. As stated in the Introduction, all subjects had extensive school experience with multiplication and division problems at least as difficult as those presented in the estimation task. The mathematicians and accountants had greater experience than the students with problem-solving in (very different) number-related domains; but probably not with tasks such as this one.

Research mathematicians, though they have almost always undergone extensive mathematical education, do not frequently practice calculations. Indeed some mathematicians strongly objected to the very term 'expert' for a mathematician. Thus, their variable and extensive strategy use may be related not only to the fact that they have access to many strategies, and to knowledge enabling them to create such strategies, but to their having a willingness and even an urge to experiment with different techniques (cf. also Krutetskii, 1981; Haylock, 1985).

Research mathematicians, unlike people who are simply skilled at computing sums, are paradoxically experts at dealing with situations where they are not experts (also cf. Schoenfield, 1986, 1987). Metaphorically they have not only learned specific arithmetical routes, but have an effective cognitive map of the territory, which makes it possible for them to take unfamiliar routes without risking losing their way here in a serious and irreversible sense. In this respect they may contrast markedly with people with limited "number sense", for whom it may be necessary to keep to known arithmetical paths if they are not to become lost. Though this study concerned mathematics, this willingness and ability to venture along unfamiliar paths is in fact likely to be a general characteristic of scientifically, artistically or otherwise 'creative' people (Bartlett, 1958).

An interesting result of the study is that the between-individual variability of the accountants in use of appropriate strategies (p.14, Table 7) was just as great as that of the mathematicians and more so than the other groups. There are at least two possible 
explanations for this finding, and it is quite possible that both are true. One is that the accountants were in fact a more heterogeneous group than the others, comprising people of a wide variety of backgrounds: most notably, educational backgrounds. This explanation is consistent with the fact that they were distinguished from the other non-mathematician groups mainly in terms of between-individual variability and not by within-individual variability (Table 6). However, it must be noted that, within the accountants' group, level of educational background did not have a marked influence on estimation strategy types (p.14). The second explanation is that their strategy variability reflects the fact that modern technology means that accountants nowadays spend little time performing mechanical calculations mentally or with pencil and paper. Calculators and computers will perform the actual arithmetical operations. The accountants' main task is to select the appropriate procedure and to interpret the results. To use Bryant's (1985) distinction, the accountants' expertise probably relates more to "knowing when to do a sum" (and which sum to use) than "knowing how to do it". Thus they may need not so much to be highly accomplished and accurate at using any particular arithmetical procedure as to be flexible in applying different arithmetical procedures in different situations.

Effects of specific aspects of mathematical experience on strategy variability need not of course be confined to such specialized groups as mathematicians and accountants: one would, for example, expect strategy variability to be influenced by the extent to which an individual's school instruction has encouraged or discouraged such variability.

\section{Nature and difficulty of the problems}

Group differences in variability may depend in part on the nature and difficulty of the particular problem. For example, there were a few exceptional problems (e.g. $648.9 \div 22.4$ ) for which mathematicians were less variable than the other groups, because they showed a strong preference for particular "Known and Nicer Numbers" strategies (e.g. in the above case 
converting the problem to $660 \div 22$ ), that were rarely accessed by the other groups. Thus it appears that while mathematical knowledge is generally associated with increased strategy variability in this task, there are indeed some problems for which mathematical knowledge and ready access to particular strategies do limit variability. Also some problems (e.g. Fermat's Last Theorem) are so difficult that even finding one solution strategy is an impressive achievement. 


\section{$\underline{\text { Appropriate versus inappropriate strategies }}$}

It is obviously important to make the distinction between the versatile use of appropriate strategies and the indiscriminate use of inappropriate ones. Variability of strategy use could be achieved by the unselective use of inappropriate and/or logically incorrect strategies (e.g. as described for young children by Dowker, 1989). Numerous researchers (e.g. Sully, 1892; Perkins, 1981; Haylock, 1985; Boden, 1990) have indeed emphasized the importance of constraints to creative problem-solving and creativity in general. (By constraints, we mean a regulatory mechanism which not only encourages the production of useful strategies but inhibits the production and use of others.)

There were striking group differences as regards the frequency of inappropriate strategy use (see Table 7). The mathematicians and accountants used very few inappropriate strategies. The actual numbers of inappropriate strategies were very similar and small in the two groups, though they were more 'popular' among the accountants than the mathematicians. The psychology students were more likely to use inappropriate strategies, but a large majority of their strategies were still appropriate. Although most of the protocols of these three groups contained inaccuracies, these were usually the result of calculation errors (cf. Dowker, 1992), or, especially in the case of the accountants and psychology students, of a readiness to accept very approximate estimates. In the case of English students, inappropriate strategies were more frequent than in the other groups (see p.14).

The findings of the present study give rise to yet further topics for research, among which are (a) to test a wider range of groups, and (b) to control more for group differences in age and professional status. In the present study, the mathematicians and accountants were mostly older than the students, and the mathematicians in particular were more advanced in their careers. Research is already in progress concerning engineers (Bagnell, 1992), mathematics undergraduates (Castell, 1992; Alexander, 1994) and academic psychologists (Malik, 1993). 


\section{ACKNOWLEDGEMENTS}

We are very grateful to the mathematicians, accountants, psychology students and English students for their kindness, patience and help. We thank Rachel Parry for her invaluable assistance with recruiting and testing English students. We are also thankful to Dr. Robert Hiorns and Alison Margetts of the Department of Statistics, Oxford University for statistical advice and help. We thank Beate Hermelin and the reviewers Arthur Baroody and Laurent Cohen for their helpful comments on earlier versions of this manuscript. The first author is grateful to the British Academy and the Economic and Social Research Council for financial support. 


\section{REFERENCES}

Alexander, P. (1994). Computational estimation strategies of mathematics undergraduates. Oxford University: Unpublished undergraduate research project.

Alpert, A. (1928). The solving of problem-situations by preschool children. Teachers College Contributions to Education, No. 23.

Bagnell, A. (1992). Computational estimation strategies of engineers. Oxford University: Unpublished undergraduate research project.

Baroody, A. J. (1987) Children's Mathematical Thinking. New York: Teacher's College Press.

Baroody, A. J. (1989). Kindergartners' mental addition with single-digit combinations. Journal for Research in Mathematics Education, 20, 159-172.

Baroody, A.J. (1992). The development of kindergartners' mental addition strategies. Learning and Individual Differences, 2(4).

Bates, E. and Elman, J. L. (1993). Connectionism and the study of change. In M. Johnson (ed.) Brain Development and Cognition: A Reader. Oxford: Basil Blackwell.

Boden, M. (1990). The Creative Mind: Myths and Mechanisms. London: Weidenfeld \& Nicolson.

Castell, N. (1992). Computational estimation strategies of undergraduate mathematicians. Oxford University: Unpublished undergraduate research project.

Defays, D. (1988). L'Esprit en Friche: Les Foisonnements de l'Intelligence Artificielle. Liege: Pierre Mardaga.

Defays, D. (1990). Numbo: a study in cognition and recognition. CC AI, 7, 217- 244.

Dehaene, S. (1992). Varieties of numerical abilities. Cognition, 44, 1-42.

Dehaene, S. and Cohen, L. (1991). Two mental calculation systems: A case study of severe acalculia with preserved approximation. Neuropsychologia, 29, 1045-1074.

Dowker, A.D. (1989, May). Computational estimation by young children. Paper presented at the Conference of the British Society for Research into Learning Mathematics, Brighton Polytechnic, Brighton, England. 
Dowker, A. D. (1992). Computational estimation strategies of professional mathematicians. Journal for Research in Mathematics Education, 23, 45-55.

Fajtlowicz, S. (1988). On conjectures of Graffiti. Discrete Mathematics, 72, 113-118.

Fajtlowicz, S. (1989, unpublished manuscript). Imperative Mathematics and Machine Intelligence.

Gill, A. J. (1993). Multiple strategies: Product of reasoning and communication. Arithmetic Teacher, 40, 380-386.

Ginsburg, H. P. (1977). Children's Arithmetic. New York: Van Nostrand.

Haylock, D.W. (1987). Mathematical creativity in schoolchildren. Journal of Creative Behaviour, 21, 48-59.

Hiebert, J. (1984). Children's mathematical learning: the struggle to link form and understanding. The Elementary School Journal, 84, 5, 497-513.

Hittmair-Delazer, M. Semenza, C. \& Denes, G. (1994). Concepts and facts in calculation. Brain, 117, 715-728.

Hittmair-Delazer, M., Sailer, U. \& Benke, T. (1995). Impaired arithmetical facts but intact conceptual knowledge: a single-case study of dyscalculia. Cortex, 31, 140-147.

Hofstadter, D.R. (1984). The Copycat project: an experiment in non-determinism and creative analogies. MIT: The Artificial Intelligence Laboratory, A.I. Memo 755

Hofstadter, D. R. (1995). Fluid Concepts and Creative Analogies: Computer Models of the Fundamental Mechanisms of Thought. New York, N.Y.: Basic Books.

Hofstadter, D.R., Mitchell, M. and French, R. M. (1987). Fluid concepts and creative analogies: a theory and its computer implementation. Indiana University Center for Research on Concepts and Cognition, Publication No.18.

Johnson-Laird, P. (1988). Freedom and constraint in creativity. In R. Sternberg (ed.) The Nature of Creativity. Cambridge: Cambridge University Press.

Karmiloff-Smith, A. (1991). Beyond modularity: innate constraints and developmental theory. In S. Carey and R. Gelman (eds.). The Epigenesis of Mind: Essays on Biology and Cognition. Hillsdale, N. J.: Lawrence Erlbaum Associates. Cambridge University Press. 
Krutetskii, V. A. (1976). The Psychology of Mathematical Abilities in Schoolchildren. Kilpatrick, J. and Wirzup, I. (eds.), Teller, J. (trans.). Chicago: University of Chicago Press.

Lefevre, J., Bisanz, J., Hubbard, K.E., Buffone, L., Greenham, S.L. and Sadesky, G.S. (Submitted). Multiple routes to solution of single-digit multiplication problems.

Lefevre, J., Sadesky, G.S. and Bisanz, J. (In press) Selection of procedures in mental addition. Reassessing the problem-size in adults. Journal of Experimental Psychology: Learning, Memory and Cognition.

Lenat, D. B. \& Brown, J. S. (1984). Why AM and EURISKO appear to work. Artificial Intelligence, 23, 269-294.

Levine, D. R. ( 1982). Strategy use and estimation of college students. Journal for Research in Mathematics Education, 13, 350-359.

Linville, P.W. and Clark, L. (1989). Can production systems cope with coping? Social Cognition, 7, 195-236.

Malik, R. (1993). Computational estimation strategies of academic psychologists. Oxford University: Unpublished undergraduate research project.

Mitchell, M. (1993). Analogy-making as Perception: A Computer Model. Cambridge, MA: M.I.T. Press.

Mitchell, M. \& Hofstadter, D.R. (1989). The role of computational temperature in a computer model of concepts and analogy-making. In Proceedings of the Eleventh Annual Conference of the Cognitive Science Society. Hillsdale, N.J.: Lawrence Erlbaum Associates.

Perkins, D. (1981). The Mind's Best Work. Cambridge, MA: Harvard University Press

Reys, R. E., Rybolt, J. F., Bestgen, B. J., \& Wyatt, J. W. (1982). Processes used by good computational estimators. Journal for Research in Mathematics Education, 13(3), 183-201.

Schoenfield, A (1986). On having and using geometric knowledge. In J. Hiebert (ed.), Conceptual and Procedural Knowledge: The Case of Mathematics. Hillsdale, N.J.: Lawrence Erlbaum Associates. 
Schoenfield, A (1987). What's all the fuss about metacognition? In A. Schoenfield (ed.) Cognitive Science and Mathematics Education. Hillsdale, N.J.: Lawrence Erlbaum Associates.

Siegler, R. S. (1987). Strategy choices in subtraction. In J. Sloboda \& D. Rogers (eds.) Cognitive Processes in Mathematics. Springer-Verlag.

Siegler, R. S. \& Jenkins, E. (1989). How Children Discover New Strategies. Hillsdale, N.J.: Lawrence Erlbaum Associates.

Smith, J.P. (1995). Competent reasoning with rational numbers. Cognition and Instruction, 13, 3-50.

Sokol, S. and McCloskey, M. (1991). Cognitive mechanisms in calculation. In R. Sternberg and P. A. Frensch: (eds.) Complex Problem Solving: Principles and Mechanisms (pp. 85-116). Hillsdale, N.J.: Erlbaum.

Sowder, J. (1992). Estimation and related topics. In D. A. Grouws (ed.) Handbook of Research on Teaching and Learning. Macmillan.

Sternberg, R. J. (1988). A three-facet model of creativity. In R. J. Sternberg (ed.) The Nature of Creativity. Cambridge: Cambridge University Press.

Sully, J. (1892). Outlines of Psychology: Revised Edition. London: Longmans, Green \& Co.

Stewart, I. (1989). Does God Play Dice? The Mathematics of Chaos. Oxford: Basil Blackwell.

Thagard, P., Holyoak, K., Nelson, G. \& Gochfield, D. (1990). Analog retrieval by constraint satisfaction. Artificial Intelligence. 46, 259-310. 